Terr. Atmos. Ocean. Sci., Vol. 18, No. 1, 85-96, March 2007

\title{
Observation of the Geocoronal Balmer Alpha with DEFPOS
}

\author{
Muhittin Sahan ${ }^{1,}{ }^{*}$, Ilhami Yegingil ${ }^{1}$, and Nazim Aksaker ${ }^{1}$ \\ (Manuscript received 11 March 2006, in final form 30 October 2006)
}

\begin{abstract}
Instrumental improvements in sensitivity of Fabry-Perot spectrometers with the development of Charged Coupled Device (CCD) cameras enable measurement of faint emission lines. A Dual Etalon Fabry-Perot Optical Spectrometer (DEFPOS) has recently been built to observe hydrogen Balmer alpha ( $\mathrm{H} \alpha: 6563 \AA$ ) emission lines from the Earth's upper atmosphere and the interstellar medium in our Galaxy. This spectrometer is now located at TUBITAK National Observatory (TUG), Antalya, Turkey, and has been observing in the zenith direction with a field-of-view of $4^{\circ} .76$ and with $1200 \mathrm{~s}$ exposure time. This paper briefly describes the DEFPOS spectrometer, data analysis, and results from data taken on 47 nights between 2002 and 2004. For the averaged geocoronal Balmer $\alpha$ intensities measured in nighttime, we found that the intensity was generally greater than $1 \mathrm{R}$ and less than 12 $R$. These first results show that there is agreement with the theory and measurements made by other groups regarding the geocoronal Balmer $\alpha$ intensities. DEFPOS observations from Turkey offer potential to combine with geocoronal hydrogen observations at other locations such as Arecibo, Puerto Rico, Wisconsin Pine Bluff Observatory (PBO), and WHAM, Kitt Peak, Arizona, enhancing our global knowledge of the upper atmosphere. In the near future, DEFPOS will be used at coudé exit of the $150 \mathrm{~cm}$ telescope. Then, it will be able to make more accurate observations at any direction in the sky.
\end{abstract}

(Key words: Geocoronal hydrogen, Techniques and methods, Fabry-Perot spectrometers )

\footnotetext{
${ }^{1}$ Department of Physics, Faculty of Sciences and Letters, University of Cukurova, Adana, Turkey

* Corresponding author address: Dr. Muhittin Sahan, Department of Physics, Faculty of Sciences and Letters, University of Cukurova, Adana, Turkey; E-mail: msahan@cu.edu.tr
} 


\section{INTRODUCTION}

The exosphere and the upper thermosphere, also called the geocorona, is the uppermost region of the earth's atmosphere characterized by low densities long, mean free paths, and non-Maxwellian velocities (Chamberlain 1987; Chamberlain and Hunten 1987). Knowledge of the geocoronal region is an important factor for improving our understanding of coupling processes between atmospheric regions. This region begins at the exobase around $500 \mathrm{~km}$ and extends out to about $80000 \mathrm{~km}$ where the earth's atmosphere is considered to merge with interplanetary space. Hydrogen, the lightest gas, is a dominant constituent of the geocorona. It was first discovered through the observations of Lyman alpha ( $\mathrm{L} \alpha: 1216 \AA$ ) radiation from a rocket flown in 1955. The hydrogen Balmer alpha ( $\mathrm{H} \alpha: 6563 \AA)$, though much fainter than Lyman- $\alpha$, can be measured from ground-based observations (Chamberlain 1987; Nossal et al. 1997). The geocoronal Balmer $\alpha$ is comprised of two fine structural components (Nossal 1994), and is produced by atomic hydrogen fluorescence following excitation by solar Lyman beta ( $\mathrm{L} \beta: 1026 \AA$ ) photons. In addition to the two directly excited Balmer $\alpha$ transitions, cascade processes excite all seven hydrogen Balmer $\alpha$ fine structure components (Kerr et al. 1986; Chamberlain 1987; Meier 1995; Nossal et al. 1998).

Different regions of the exosphere can be probed by measuring the line of sight column emission as the earth's shadow height changes during the night. The shadow height is a function of time and viewing geometry. For instance, if a zenith viewing geometry is maintained, the shadow height will be lower at twilight and higher towards midnight. Thus, while the entire exosphere is illuminated at twilight, only the progressively higher altitude portions of it are so towards midnight. Most of the emission, especially at low shadow height, comes from above the shadow, where the geocorona is illuminated directly by the solar Lyman radiation (Shih et al. 1985; Nossal et al. 1993, 1997, 1998).

The first observations of the geocoronal Balmer $\alpha$ emission in the night sky began in 1957 at Zvenigorod in the Soviet Union, although the source of the emission was not clear at that time (Krassovsky and Galperin 1958). Moreover, the first observations of the $\mathrm{H} \alpha$ spectral profile at high resolution were measured using a Fabry-Perot interferometer of low sensitivity from Arecibo observatory in 1971 - 1972 (Atreya et al. 1975) and later in 1980 (Meriwether et al. 1980; Kerr et al. 1986; Kerr and Hecht 1996) at Arecibo Observatory, Puerto Rico. The geocoronal Balmer $\alpha$ emission line profile has also been regularly measured at the University of Wisconsin at Pine Bluff Observatory (PBO), near Madison since the 1970s (Reynolds et al. 1973; Shih et al. 1985; Yelle and Roesler 1985; Nossal et al. 1997; Mierkiewicz 2002). A group of researchers, led by Reynolds, Roesler and Scherb, from the University of Wisconsin, developed a new remotely operated project called WHAM (Wisconsin Hydrogen Alpha Mapper), which is now located at Kitt Peak National Observatory, Arizona (Tufte 1997; Haffner et al. 2003). With WHAM, over $37000 \mathrm{H} \alpha$ spectra have been obtained from the interstellar medium since 1997. Although WHAM is used primarily for making astronomical observations of the interstellar medium emission (Haffner et al. 2003), the terrestrial emissions present in the WHAM astronomical Balmer $\alpha$ spectra offer a rich resource for studying the geocorona. The continuing WHAM H $\alpha$ observations should provide solid measures of the geocoronal $\mathrm{H} \alpha$ emission (Nossal et al. 2001). 
Consequently, Fabry-Perot observations have been made continuously both at the Arecibo and Wisconsin sites since the early 1980s (Meriwether et al. 1980; Shih et al. 1985; Yelle and Roesler 1985; Kerr et al. 1986; Kerr and Hecht 1996; Nossal et al. 1998, 2001). Similar to these two groups, we have designed and built the DEFPOS (Dual Etalon Fabry-Perot Optical Spectrometer) to measure Balmer $\alpha$ emission from the geocorona and the interstellar medium of our Galaxy (Sahan et al. 2005). In this paper, we introduce the spectrometer itself and present the very first results of geocoronal measurements obtained with it.

\section{INSTRUMENT AND DATA ANALYSIS}

Since Fabry-Perot spectrometers are capable of simultaneously achieving high spectral resolution and high throughput, they are well suited for detailed studies of faint diffuse emissions such as the geocorona and the interstellar medium (Roesler 1974; Nossal 2001). Therefore, a 75-mm, dual etalon Fabry-Perot optical spectrometer was developed in order to measure hydrogen Balmer $\alpha$ emission from the geocorona and the interstellar medium of our Galaxy. We named this spectrometer as DEFPOS (Dual Etalon Fabry-Perot Optical Spectrometer) and set it up at TUBITAK National Observatory (TUG) at Bakirlitepe $\left(36^{\circ} 51^{\prime} \mathrm{N} ; 30^{\circ} 20^{\prime} \mathrm{E}\right.$; elevation: $2547 \mathrm{~m}$ ) near Antalya, Turkey. Figure 1 shows the usual optical arrangement of the DEFPOS spectrometer. As seen from Fig. 1, the spectrometer includes a narrow-band interference filter ( $15 \AA$ FWHM) centered around $\mathrm{H} \alpha(6563 \AA)$, two Fabry-Perot etalons, and a focusing lens of focal length of $30 \mathrm{~cm}$. Since the spectrometer observes directly in the zenith with a $4^{\circ} .76$ circular

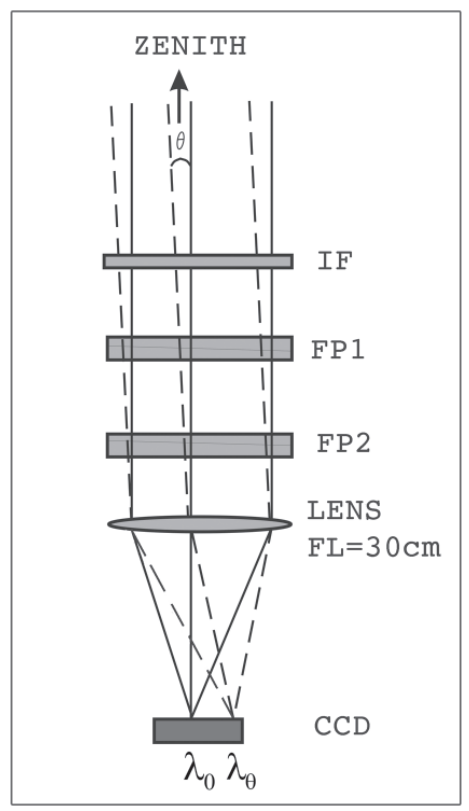

Fig. 1. Basic optical layout of the DEFPOS spectrometer. Light from zenith direction with a field-of-view of $4^{\circ} .76$ enters through a filter first, and then through FP1 and FP2, respectively. The $30 \mathrm{~cm}$ focusing lens forms an image of the spectra onto the CCD camera. 
field of view (FOV), the light beam firstly passes through the interference filter, and then through the first etalon (FP1), then the second (FP2), which enhances the instrumental contrast between the peak and the background of the instrumental profile. The second etalon also suppresses ghost emissions, and reduces parasitic light contamination (Roesler 1974). Then, light passes through the imaging lens $(\mathrm{fl}=30 \mathrm{~cm})$, and finally is focused as a ring image onto $\mathrm{CCD}$, which has a size of $3 \times 3 \mathrm{~cm}^{2}$ with $15 \mu \mathrm{m}$ square pixels (Yegingil et al. 2004; Sahan et al. 2005). Further details of the spectrometer are described in Sahan et al. (2005).

In order to accurately convert a CCD image into a line profile, a series of image processing procedures is required. We used a standard data reduction technique for CCD frames to obtain pure $\mathrm{H} \alpha$ spectra from the CCD images. This technique includes cosmic-ray removal, dark and bias subtraction, and flat-fielding as discussed previously in Sahan et al.'s paper (2005). The reduced $\mathrm{H} \alpha$ images were converted into the line profiles by summing the intensities of the pixels lying in equal area annular bins of increasing radius from the ring center (Coakley and Roesler 1994; Coakley et al. 1996; Sahan 2004). This technique is based upon the property that equal area annuli correspond to equal spectral intervals on the Fabry-Perot ring pattern (Coakley et al. 1996; Nossal et al. 1997).

The observed Balmer $\alpha$ profiles are approximately of Gaussian shapes and include Galactic and geocoronal $\mathrm{H} \alpha$ components (see Fig. 2a). In general, the separation between the interstellar emission and the geocoronal line is due to a combination of earth's orbital velocity, the sun's peculiar velocity, and intrinsic motions of the interstellar gas, including Galactic differential rotation (Reynolds et al. 1998). It is necessary to separate the geocoronal and Galactic $\mathrm{H} \alpha$ emission lines from each other. Therefore, we have used a curve fitting program to fit Gaussians to each observed spectrum. Firstly, we have determined the geocoronal Balmer $\alpha$ component of the spectrum. Thus, a Gaussian line shape has been fitted to each spectrum using a program that determines the intensity of each geocoronal emission line (Nossal et al. 1993). For the geocoronal line, only one Gaussian was used for all cases. Using this fitting program, we have determined amplitudes, locations, and full width half maximum (FWHM) of all individual line profiles. The location of the geocoronal hydrogen line in each spectrum has been determined by comparison to the examination of the spectral line profile of a hydrogen lamp (Meriwether et al. 1980). So, the position and the approximate line width (FWHM) of the geocoronal line have been determined. For each individually observed Galactic line, one or more Gaussians is/are needed with different widths and area to get the most appropriate fit. Areas of the Gaussians have been then used to determine the intensity of both the geocoronal and the Galactic $\mathrm{H} \alpha$ lines. Galactic lines have then been removed from the data by fitting each spectrum with Gaussian components and subtracting the Galactic Gaussian from the original spectrum (e.g., Fig. 2b).

For calibration of intensity, it is necessary to maintain long term source calibration stability. Nebular calibration sources are suitable for such stability. These sources are diffuse, and they fill the Fabry-Perot's FOV. Moreover, since these sources are outside of the Earth's atmosphere, they are subject to the same atmospheric extinction as the geocorona. Therefore, the WHAM spectrometer has used observations of the North American Nebula (NAN; $850 \pm 50 \mathrm{R} \mathrm{H} \alpha$ ) for calibration, where 1 Rayleigh (R) is $10^{6} 4 \pi^{-1}$ photons $\mathrm{cm}^{-2} \mathrm{sr}^{-1} \mathrm{~s}^{-1}=2.41 \times 10^{-7} \mathrm{erg} \mathrm{cm}^{-2} \mathrm{~s}^{-1} \mathrm{sr}^{-1}$ at $\mathrm{H} \alpha$ (Reynolds 1985; Reynolds et al. 1999; Haffner et al. 2003). But, since DEFPOS has not 
been used with a telescope or a sidereostat in the same manner as WHAM, it is impossible to observe any direction other than zenith. Therefore, as explained by Sahan et al. (2005), we have used the WHAM $\mathrm{H} \alpha$ data with the same region to calibrate $\mathrm{H} \alpha$ intensity in Rayleigh unit (Sahan et al. 2005). Note that we obtained the WHAM data from the web site of $w w w$. wham.astro.wisc.edu/wham.

\section{RESULTS AND DISCUSSION}

As mentioned in the previous section, DEFPOS has been designed to observe faint optical Balmer $\alpha$ line emission from the diffuse ionized hydrogen gas in our Galaxy. It has been designed to be used at the coudé focus of the $150 \mathrm{~cm}$ telescope installed at TUG, but since the coudé exit of the telescope was not ready at that time, the spectrometer was set up in a room on the upper floor of the telescope building, and observations began in the zenith direction in October 2002. At this location, DEFPOS has no pre-etalon optics; it looks at a large FOV of $4^{\circ} .76$ and sweeps $9^{\circ}$ in 1200 seconds of integration time. So, during this time, the effective FOV of the instrument is $4^{\circ} .76 \times 9^{\circ}$ on the sky. Between October 2002 and December 2004, we obtained $476 \mathrm{H} \alpha$ spectra on 47 nights. For all the data, 1200 second integrations were used in order to achieve a signal to noise $(\mathrm{S} / \mathrm{N})$ ratio greater than 10 for the Galactic $\mathrm{H} \alpha$ line, whose brightness is $0.50 \mathrm{R}$. Since the resolution of DEFPOS is 11000 , which corresponds to instrumental line width of $27 \mathrm{~km} \mathrm{~s}^{-1}$, and the line width of the geocoronal $\mathrm{H} \alpha$ line is around $7 \mathrm{~km} \mathrm{~s}^{-1}$, it is impossible to determine the temperature of the geocoronal hydrogen line with this resolution.

Since DEFPOS has observed the Galactic $\mathrm{H} \alpha$ through the earth's atmosphere, all observed spectra have included the atmospheric Balmer $\alpha$ emission line besides the Galactic line. In order to examine the geocoronal Balmer $\alpha$ emission line, Galactic lines have been extracted from each individual spectrum. In order to determine the position of the geocoronal line, the Local Standard of Rest (LSR) has been calculated and the position of the atmospheric line is taken as $-\mathrm{V}_{\mathrm{LSR}}-2.33 \mathrm{~km} \mathrm{~s}^{-1}$ (Haffner et al. 2003), and this way, the positions of the Galactic lines are thus determined (Fig. 2). The $-2.33 \mathrm{~km} \mathrm{~s}^{-1}(0.05 \AA)$ correction is necessary due to difference in the rest wavelengths of the geocoronal lines, which consist of two fine structure transitions, and the galactic recombination line at $6562.82 \AA$ which has all seven of the transmission contributing. All Galactic emission lines have been removed from the spectra. This procedure is well illustrated in Fig. 2 below. The processed data given in Fig. 2 were taken on July 29 - 30, 2003 during 0020 to 0040 UT (Universal Time). The center coordinate of the observed region is $b=-20^{\circ} .90$ and $l=99^{\circ} .40$. Since indication of Doppler shifts between galactic and the geocoronal emission is very useful in velocity unit, spectral displacement on the horizontal axis were expressed here in velocity units $\left(1 \mathrm{~km} \mathrm{~s}^{-1}\right.$ corresponds to $0.0219 \AA$ at $\mathrm{H} \alpha)$ and galactic and geocoronal $\mathrm{H} \alpha$ components are observed at $260 \mathrm{~km} \mathrm{~s}^{-1}(\sim 5.68 \AA)$ spectral window. Zero velocity is VLSR velocity on each plot. Galactic and geocoronal $\mathrm{H} \alpha$ lines are shown as dotted lines. The prominent and narrow emission component denoted as 2 at $26.44 \mathrm{~km} \mathrm{~s}^{-1}$ is the geocoronal $\mathrm{H} \alpha$ emission produced by the scattering of the solar Lyman- $\beta$ radiation within the geocorona. Its width in the spectrum is about $30 \mathrm{~km} \mathrm{~s}^{-1}$, which is determined by the resolution of the spectrometer. Since our Fabry-Perot spectrometer has an optical field-of-view on the sky of $\sim 4^{\circ} .76$, and a spectral range at Balmer $\alpha$ of $\sim 260 \mathrm{~km} \mathrm{~s}^{-1}(\sim 5.68 \AA)$ 
with $27 \mathrm{~km} \mathrm{~s}^{-1}(\sim 0.59 \AA)$ spectral resolution ( $\left.\mathrm{R}=\lambda / \Delta \lambda \sim 11000\right)$, DEFPOS is not capable of resolving the $\sim 7 \mathrm{~km} \mathrm{~s}^{-1}$ (FWHM) geocoronal Balmer $\alpha$ line profile (Meriwether et al. 1980; Yelle and Roesler 1985). The intensity of the geocoronal Balmer $\alpha$ at this time was around 4.78 R. In addition to the geocoronal $\mathrm{H} \alpha$ emission line, there are two weaker features from two ionized interstellar hydrogen clouds along this line of sight (Haffner et al. 2003). These Galactic features were denoted as 1 on the left/blue side, and as 3 on the right/red side of the geocorona
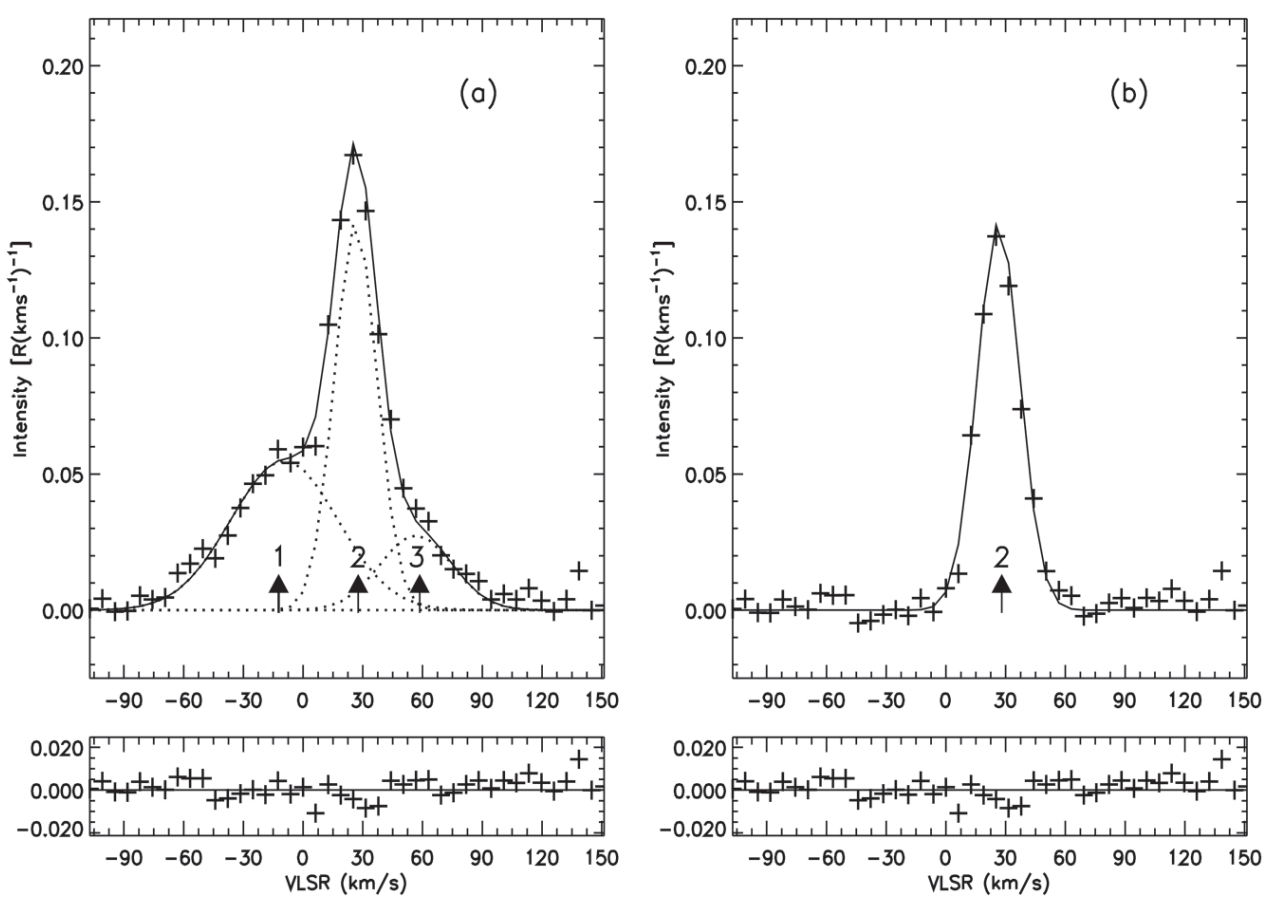

Fig. 2. A spectrum obtained on July 30, 2003 around 0030 UT. The center coordinate of the observed region is $b=-20^{\circ} .9$ and $l=99^{\circ} .40$. In Fig. 2a, there is a prominent feature (2) which is the geocoronal $\mathrm{H} \alpha$ line and there are two Galactic features $(1,3)$ from two ionized interstellar hydrogen clouds along this line of sight (Haffner et al. 2003). It can clearly be seen that the geocoronal emission line is superimposed on the two Galactic emission lines. The intensity of the geocoronal line is $4.78 \mathrm{R}$. Its width in the spectrum is about $30 \mathrm{~km} \mathrm{~s}^{-1}$, and is determined by the resolution of the spectrometer. The weaker features, on the left denoted as 1 and on the right as 3, are Galactic emission lines and their intensities are $3.22 \mathrm{R}$, and $1.30 \mathrm{R}$, respectively, Fig. 2b. The resulting pure geocoronal $\mathrm{H} \alpha$ spectrum after removal of the Galactic $\mathrm{H} \alpha$ lines is shown in Fig. $2 b$. The residuals of each fit are plotted at the bottom of the figures. 
line. The intensity values of these features are $3.22 \mathrm{R}$ at $-15.40 \mathrm{~km} \mathrm{~s}^{-1}$ for the left side, and $1.30 \mathrm{R}$ at $60.81 \mathrm{~km} \mathrm{~s}^{-1}$ for the right side. After these two Galactic $\mathrm{H} \alpha$ components were removed from the spectrum, the pure geocoronal $\mathrm{H} \alpha$ spectrum was obtained (Fig. 2b). The residuals of each fit are also plotted at the bottom of the figures.

The intensity of the geocoronal Balmer $\alpha$ emission typically ranges from $\sim 1-25 \mathrm{R}$, and it depends most sensitively on the height of the earth's shadow along the direction of the observation. The range of the solar depression angle covered by a fixed observing site throughout the year is dictated by the local latitude. With a latitude of $36^{\circ} .82^{\prime} \mathrm{N}$, which is the latitude of the TUG observatory, the largest depression angles occur at local midnight where it varies from $23^{\circ} .47$ at summer solstice to $60^{\circ} .37$ at the winter solstice (Shih et al. 1985). The data obtained during the summer nights are usually limited to a smaller range of solar depression angles than during the winter nights. At TUG, the solar depression angle in June ranges approximately from $10^{\circ}$ at the solar twilight to $30^{\circ}$ at solar midnight, while in December it ranges between about $20^{\circ}$ at twilight and $75^{\circ}$ at midnight (see Fig. 3). Thus geocoronal $\mathrm{H} \alpha$ intensity values are limited approximately to $10^{\circ}$ to $30^{\circ}$ at twilight in the summer (see Fig. 4). In the zenith direction, since the declination is constant and equal to the latitude of the observatory, the intensity of the geocoronal line changes from a minimum at midnight to a maximum at twilight.

A computer program was prepared to compute the shadow height and solar depression angle. Examples of the intensity variation of the geocoronal line with shadow height and solar depression angle are given in Figs. 3a and b, respectively. These geocoronal Balmer $\alpha$ data were obtained on the night of September $27-28,2003$. A total of $25 \mathrm{H} \alpha$ spectra were obtained during this observation night and each observation was illustrated with filled triangles in Fig. 3b and also illustrated with open triangles in Fig. 4. The solar right ascension toward the zenith is approximately $00^{h} .32$ and the declination is $36^{\circ} .82$, which is the constant latitude of the observation because of zenith orientation. The shadow height (solar depression angle) changes approximately from $462 \mathrm{~km}\left(15^{\circ}\right)$ to $4813 \mathrm{~km}\left(58^{\circ}\right)$ during this time interval. With this observational geometry, the shadow height, and hence the intensities change faster at twilight than near midnight. The variation of the shadow height and solar depression angle are obviously similar, and the variation of the intensity of the atmospheric $\mathrm{H} \alpha$ line decreases with increasing shadow height as expected. The decrease in $\mathrm{H} \alpha$ intensity with increasing solar depression angle is consistent with resonant fluorescent sources, since the height of the base of the illuminated column increases with increasing solar depression angle. As clearly seen from Fig. 3, the intensity of the geocoronal line changed from $1.50 \mathrm{R}$ (at midnight) to $11.13 \mathrm{R}$ (at twilight) depending on the solar shadow height. In Fig. 3a, star and plus symbols represent solar depression angle and shadow height as a function of UT, respectively.

Geocoronal Balmer $\alpha$ intensity data obtained between October 2002 and December 2004 are plotted in Fig. 4 as a function of the solar depression angle at TUG observatory for a collection of dates when the best data was obtained. The observations obtained before and after midnight UT are plotted on the same figure. Some data in this plotting were excluded, because they were remarkably different from all other nights at which we made observations and therefore indicate some as yet unidentified problem. Due to the faintness of geocoronal Balmer $\alpha$ emission, observations were made only when the solar depression angle was greater 
than $10^{\circ}$, otherwise the geocoronal Balmer $\alpha$ can be easily overwhelmed by the sky light background. The variations in Balmer $\alpha$ intensities in Fig. 4 are associated with line of sight variations in the earth's shadow height. As the solar depression angle increases into the more diffuse regions of exosphere, less hydrogen is directly illuminated by solar Lyman- $\beta$ radiation and the emission intensity decreases. In general, the morning geocoronal Balmer $\alpha$ brightness is greater than evening brightness for solar depression angle below about $40^{\circ}$. This maximum hydrogen brightness in the early morning was firstly identified by Tinsley (1975). For the averaged geocoronal $\mathrm{H} \alpha$ intensities measured during the nighttime, we found that the intensity was generally greater than $1 \mathrm{R}$ and less than $12 \mathrm{R}$. The observed slight increase of the morning geocoronal $\mathrm{H} \alpha$ emission value over the corresponding evening value tends to agree
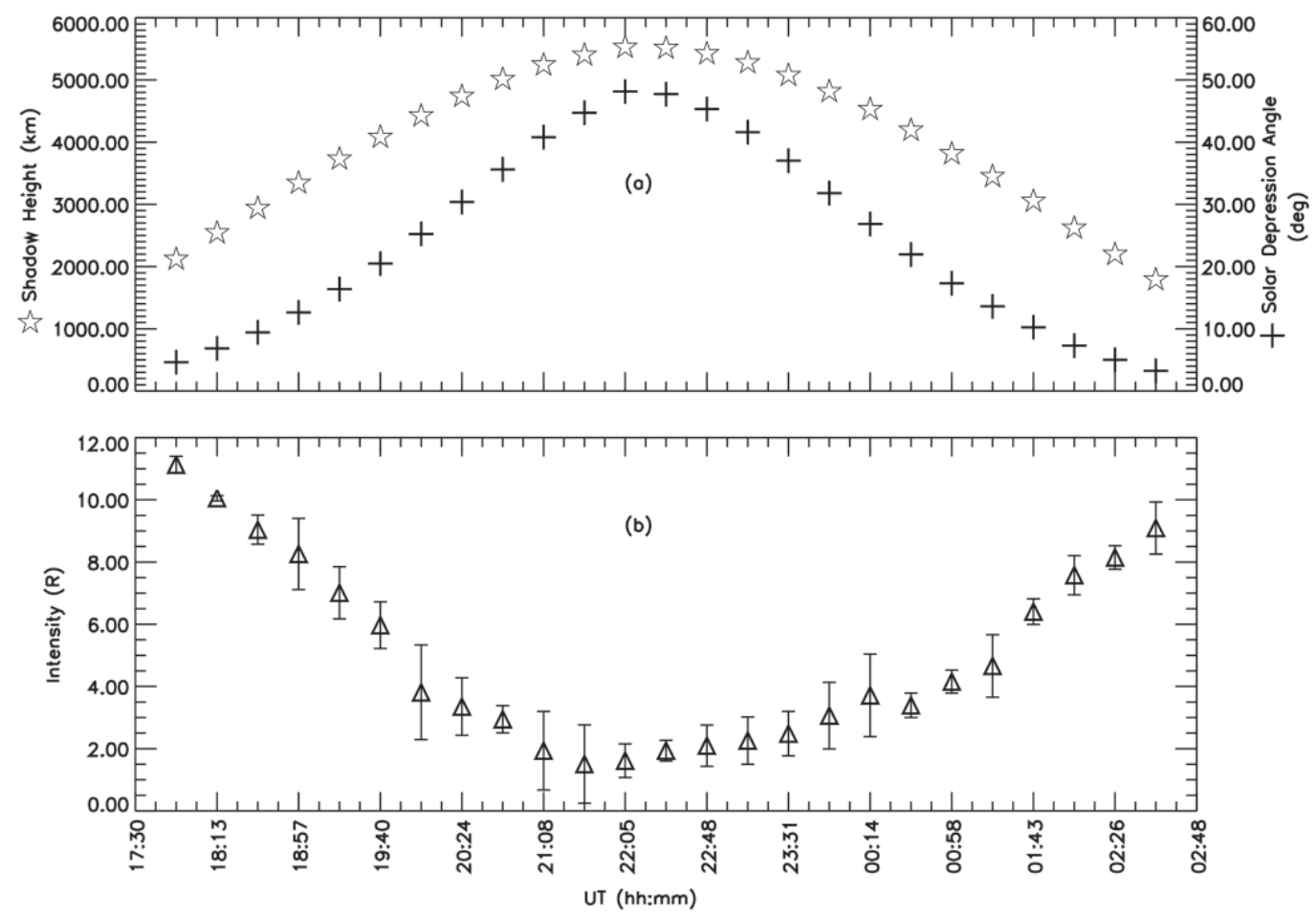

Fig. 3. The data obtained on the night of September 27 - 28, 2003 showing: (a) variation of solar depression angle (star), shadow height (plus); and (b) intensity of atmospheric $\mathrm{H} \alpha$ (triangle). The solar right ascension toward the zenith is approximately $00^{h} .32$ and the declination is $36^{\circ} .82$ which is the constant latitude of the observation because of the instrument's zenith orientation. The shadow height changes approximately from $462 \mathrm{~km}$ to 4813 $\mathrm{km}$ during this time interval. With this observation geometry, the shadow height, and hence the intensities, change faster at twilight than near midnight. 


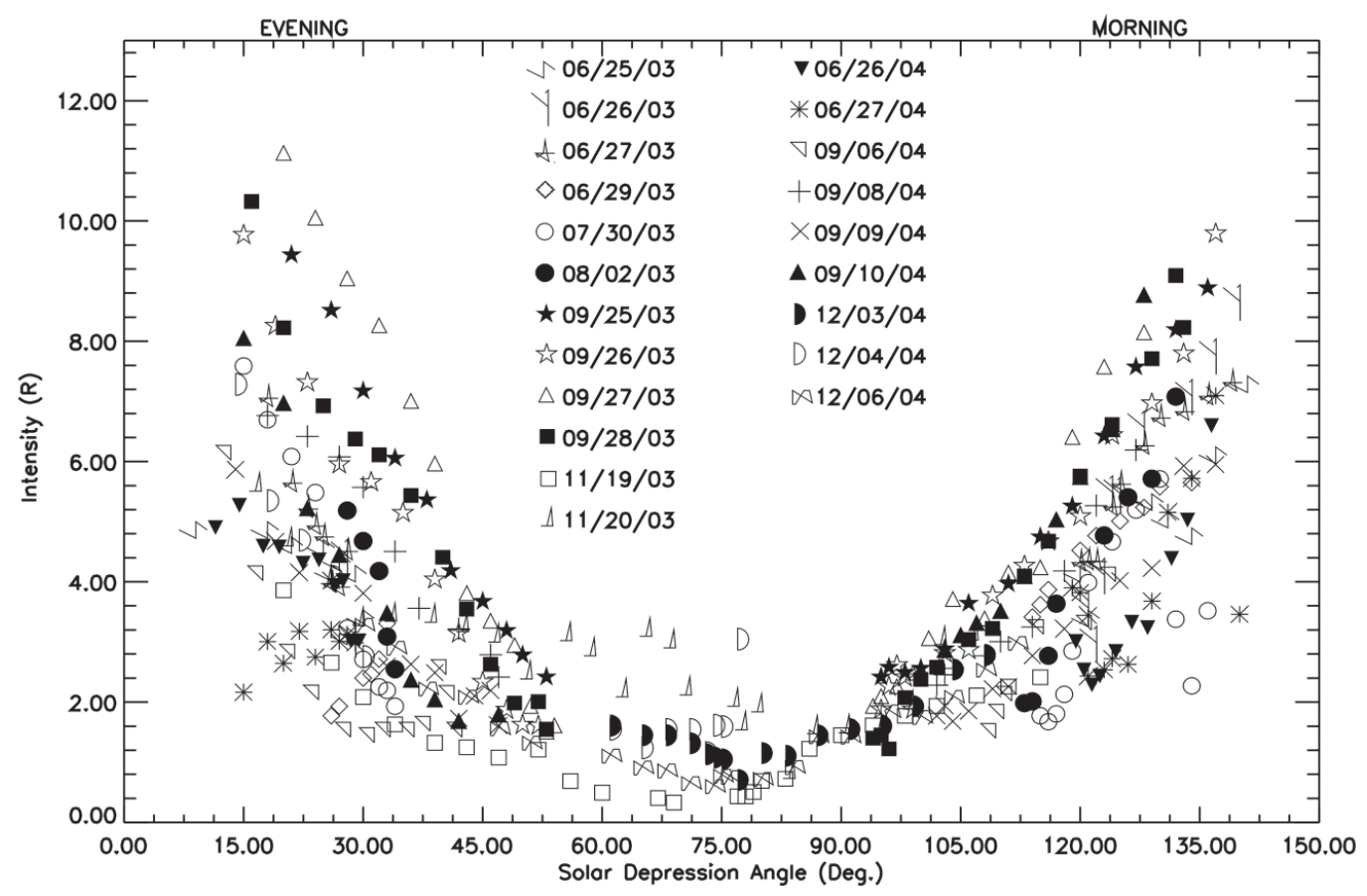

Fig. 4. The relative Geocoronal Balmer $\alpha$ intensity variation versus solar depression angle. The observations were taken looking in the zenith direction. Geocoronal Balmer $\alpha$ intensity values vary between $0.5 \mathrm{R}$ and $12 \mathrm{R}$.

with the nighttime hydrogen distribution which shows no great asymmetry before and after midnight (Chamberlain 1987; Chamberlain and Hunten 1987). These first results obtained by DEFPOS generally show that there is an agreement with the theory and earlier measurements of $\mathrm{H} \alpha$ intensities. As seen from Fig. 4, some of the spread data from night to night may be due to differences in sky conditions, including some nights with partial cloud cover.

\section{CONCLUDING REMARKS}

The large enhancement in the sensitivity of the Fabry-Perot spectrometer opened up a new window for ground-based measurements of Galactic and geocoronal $\mathrm{H} \alpha$ measurements. The observations which led to such measurements carried out by scholars in the field greatly contributed to our understanding of the diurnal, seasonal, and solar cycle variations of the geocoronal $\mathrm{H} \alpha$ intensity. One new Fabry-Perot for making such observations is DEFPOS, which we have designed to observe faint optical Balmer $\alpha$ line emissions from the diffuse ionized gas in our Galaxy and the uppermost region of the earth's atmosphere (geocorona) 
used in this research. Measurements of geocoronal and Galactic Balmer $\alpha$ emission lines were made between 2002 and 2004 with a large FOV of $4^{\circ} .76$ and with $1200 \mathrm{~s}$ exposure times in the zenith direction. These results demonstrate that our spectrometer is suitable for geocoronal and Galactic $\mathrm{H} \alpha$ measurements. In the near future, DEFPOS will be used at coudé exit of the $150 \mathrm{~cm}$ telescope located at TUG. The FOV of the spectrometer then will be about 4 arcmin. With this low angular resolution, DEFPOS will be used to investigate some of the interesting HII regions and some filaments in our Galaxy seen on the WHAM map. Besides interstellar studies, DEFPOS will be used to observe the geocorona to examine the state of hydrogen and its variation at any direction in the sky. Long term measurement of geocoronal $\mathrm{H} \alpha$ intensities can also be used to predict long term trends in variation of the greenhouse gas in the lower atmosphere (Roble and Dickinson 1989). DEFPOS observations from Turkey offer the potential of combining such observations with geocoronal hydrogen observations at other locations such as Arecibo Observatory, Puerto Rico, Wisconsin Pine Bluff Observatory (PBO), and Kitt Peak, Arizona, enhancing our global knowledge of the upper atmosphere.

Acknowledgements The authors wish to thank R. J. Reynolds, F. L. Roesler, L. M. Haffner, and S. M. Nossal, and E. J. Mierkiewicz from Wisconsin University for letting us use their data and for having greatly contributed to the formation of this study. The work documented in this paper was supported by Cukurova University, Middle East Technical University (METU), Sabanci University, and TUG. Our indebtedness goes to all members of these institutions who did their best providing us with all the necessary technical and logistic support. We also thank Umit Kiziloglu from METU, for his invaluable contribution and assistance at various times during the design, construction, and the testing phases of DEFPOS. This work is now supported by TUBITAK (The Scientific and Technical Research Council of Turkey) with grant number $104 \mathrm{~T} 252$.

\section{REFERENCES}

Atreya, S. K., P. B. Hayes, and A. F. Nagy, 1975: Doppler profile measurements of the geocoronal hydrogen Balmer alpha line. J. Geophys. Res., 80, 635-638.

Chamberlain, J. W., 1987: Balmer profiles in the geocorona and interstellar space, I. Asymmetries due to fine structure. Icarus, 70, 476-482.

Chamberlain, J. W., and D. M. Hunten, 1987: Theory of planetary atmospheres: an introduction to their physics and chemistry, $2^{\text {nd }}$ Ed., Academic Press, Inc., California, 330-415.

Coakley, M. M., and F. L. Roesler, 1994: Application of conventional CCD cameras with Fabry-Perot spectrometers for airglow observations. Proc. SPIE Int. Soc. Opt. Eng., 2266, 122-132.

Coakley, M. M., F. L. Roesler, R. J. Reynolds, and S. Nossal, 1996: Fabry-Perot/CCD annular summing spectroscopy: study and implementation for aeronomy applications. Appl. Optics, 35, 6479-6493.

Haffner, L. M., R. J. Reynolds, S. L. Tufte, G. J. Madsen, K. P. Jaehnig, and J. W. Percival, 2003: The Wisconsin H $\alpha$ Mapper northern sky survey. Astrophys. J. Suppl. Ser., 149, 405-422. 
Kerr, R. B., S. K. Atreya, J. W. Meriwether, C. A. Tepley, and R. G. Burnside, 1986: Simultaneous $\mathrm{H} \alpha$ line profile and radar measurements at Arecibo. J. Geophys. Res., 91, 4491-4512.

Kerr, R. B., and J. H. Hecht, 1996: Measurement of geocoronal Balmer alpha in the auroral zone. J. Geophys. Res., 101, 189-194.

Krassovsky, V. I., and G. I. Galperin, 1958: Trans. Int. Astr. Union, 10, 367 pp.

Meier, R. R., 1995: Solar Lyman series line profiles and atomic hydrogen excitation rates. Astrophys. J., 452, 462-471.

Meriwether, J. W., S. K. Atreya, T. M. Donahue, and R. G. Burnside, 1980: Measurements of the spectral profile of Balmer alpha emission from the hydrogen geocorona. Geophys. Res. Lett., 7, 967-970.

Mierkiewicz, E. J., 2002: Fabry-Perot observations of the hydrogen geocorona. Ph. D. Thesis, University of Wisconsin, Madison, USA.

Nossal, S., F. L. Roesler, R. J. Reynolds, and F. Scherb, 1993: Solar cycle variations of geocoronal Balmer alpha emission. J. Geophys. Res., 98, 3669-3676.

Nossal, S., 1994: Fabry-Perot observations of geocoronal hydrogen Balmer alpha emissions. $\mathrm{Ph}$. D. Thesis, University of Wisconsin, Madison, USA.

Nossal, S., F. L. Roesler, M. M. Coakley, and R. J. Reynolds, 1997: Geocoronal hydrogen Balmer $\alpha$ line profiles obtained using Fabry-Perot annular summing spectroscopy: effective temperature results. J. Geophys. Res., 102, 14541-14553.

Nossal, S., F. L. Roesler, and M. M. Coakley, 1998: Cascade Excitation in the geocoronal hydrogen Balmer $\alpha$ line. J. Geophys. Res., 103, 381-390.

Nossal, S., F. L. Roesler, R. J. Reynolds, M. Haffner, S. Tufte, J. Bishop, and J. Percival, 2001: Geocoronal Balmer $\alpha$ intensity measurements using the WHAM Fabry-Perot facility, J. Geophys. Res., 106, 5605-5616.

Reynolds, R. J., F. L. Roesler, and F. Scherb, 1973: Low intensity balmer emissions from the interstellar medium and geocorona. Astrophys. J., 179, 651-657.

Reynolds, R. J., 1985: Detection of a large very faint emission nebula surrounding Alpha Virginis. Astron. J., 90, 92-94.

Reynolds, R. J., S. L. Tufte, L. M. Haffner, K. Jeahnig, and J. W. Percival, 1998: The Wisconsin $\mathrm{H}$ alpha mapper (WHAM): a brief review of performance characteristics and early scientific results. Publ. Astron. Soc. Aust., 15, 14-18.

Reynolds, R. J., L. M. Haffner, and S. L. Tufte, 1999: The Gaseous ISM: observations with the Wisconsin H-alpha mapper (WHAM). ASP Conf. Ser., 168, 149-160.

Roble, R. G., and R. E. Dickinson, 1989: How will changes in carbon dioxide and methane modify the mean structure of the mesosphere and thermosphere. Geophys. Res. Lett., 16, 1441-1444.

Roesler, F. L., 1974: Fabry-Perot instruments for astronomy, in Methods of Experimental Physics. In: Carlton, N. P. (Ed.), 12, Part A, Academic Press Inc., New York, 531 pp.

Sahan, M., 2004: DEFPOS: design, performance characteristics and first results. Ph. D. Thesis, University of Cukurova, Adana, Turkey.

Sahan, M., I. Yegingil, U. Kiziloglu, N. Aksaker, and M. Akyilmaz, 2005: DEFPOS and its first results. Chin. J. Astron. Astrophys., 5, 211-220. 
Shih, P., F. L. Roesler, and F. Scherb, 1985: Intensity variations of geocoronal Balmer alpha emission: I-Observational results. J. Geophys. Res., 90, 477-490.

Tinsley, B. A., R. R. Hodges, and D. F. Strobel, 1975: Diurnal variations of atomic hydrogen: observations and calculations. J. Geophys. Res., 80, 626-634.

Tufte, S. L., 1997: WHAM Spectrometer: design, performance characteristics and first results. $\mathrm{Ph}$. D. Thesis, University of Wisconsin, Madison, USA.

Yegingil, I., U. Kiziloglu, M. Sahan, M. Akyilmaz, and N. Aksaker, 2004: DEFPOS (Dual Etalon Fabry-Perot Optical Spectrometer). In: Uyaniker, B., W. Reich, R. Wielebinski (Eds.), The Magnetized Interstellar Medium, Proceedings of the conference, Turkey, 227-231.

Yelle, R. V., and F. L. Roesler, 1985: Geocoronal Balmer alpha line profiles and implications for he exosphere. J. Geophys. Res., 90, 7568-7580.

Sahan, M., I. Yegingil, and N. Aksaker, 2007: Observation of the geocoronal Balmer alpha with DEFPOS. Terr. Atmos. Ocean. Sci., 18, 85-96. 